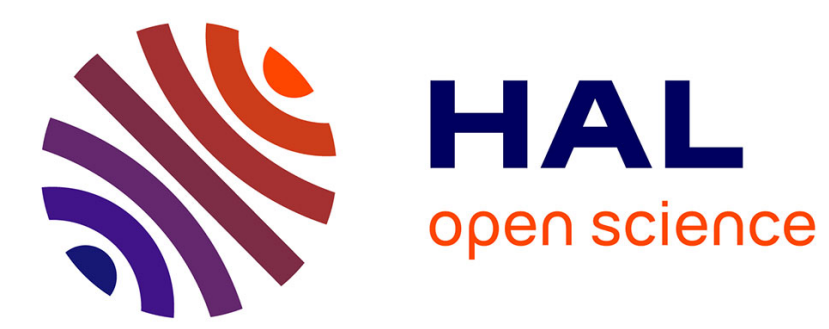

\title{
Éditer la Révélation
}

Anouk Cohen

\section{To cite this version:}

Anouk Cohen. Éditer la Révélation. Genèses. Sciences sociales et histoire, 2016, 105 (105), pp.57-75.

10.3917/gen.105.0057 . hal-01632140

\section{HAL Id: hal-01632140 \\ https://hal.parisnanterre.fr/hal-01632140}

Submitted on 15 Dec 2020

HAL is a multi-disciplinary open access archive for the deposit and dissemination of scientific research documents, whether they are published or not. The documents may come from teaching and research institutions in France or abroad, or from public or private research centers.
L'archive ouverte pluridisciplinaire HAL, est destinée au dépôt et à la diffusion de documents scientifiques de niveau recherche, publiés ou non, émanant des établissements d'enseignement et de recherche français ou étrangers, des laboratoires publics ou privés. 


\title{
Éditer la Révélation
}

\section{Le Coran dans le Maroc contemporain}

\author{
Anouk Cohen
}

e que nous vendons le mieux est le Coran, il est importé dans
des quantités énormes. J'en reçois dix mille exemplaires tous les
deux ou trois mois et je les vends facilement!» s'exclame Fouad Korrich, propriétaire d'une librairie de cinq salariés dans le quartier des Habous, centre du livre arabe à Casablanca. On assiste depuis le milieu des années 2000 à une explosion du marché coranique au Maroc. En témoigne l'ouverture récente de boutiques spécialisées dans les livres religieux à Casablanca, la capitale économique du pays. Ce «boom» du livre saint est lié à de profondes transformations de la société telles que les progrès d'alphabétisation, l'urbanisation, l'élévation du niveau de vie ou le nombre croissant d'écoles coraniques financées par le parti islamiste au pouvoir (Parti de la justice et du développement). Ces changements étendent l'accès du livre saint à de nouvelles catégories de la population - notamment les femmes, de plus en plus alphabétisées, les jeunes scolarisés, et les analphabètes, grâce au Coran audio (Cohen 2016). Le Coran est devenu en quelques années un véritable "best seller», comme le qualifient les libraires, et certains d'entre eux ont commencé à éditer leur propre modèle de livre saint. Le présent article s'appuie sur une enquête ethnographique réalisée dans le quartier des Habous entre 2007 et 2010, soit juste avant la reprise en main de ce marché par l'État grâce à la création de la Fondation Mohammed VI pour l'édition du saint Coran, «compétente à titre exclusif pour procéder aux opérations d'enregistrement, d'impression, de publication et de distribution du Livre saint ${ }^{1} »$. L'enquête saisit ainsi une période marquée par une forte concurrence entre éditeurs marocains et aussi entre éditeurs marocains et éditeurs égyptiens, libanais ou saoudiens.

$\mathrm{Au}$ Maroc, comme ailleurs, l'éditeur à succès est celui qui sait adapter son catalogue à ses publics variés, majoritairement caractérisés par un faible pouvoir d'achat. Le prix du livre saint, compris entre 3 dirhams (environ 25 cents d'euros) et 500000 dirhams (45000 euros) pour les modèles les plus sophistiqués, est 
déterminant dans la réussite commerciale. Les éditeurs tentent de le réduire en diminuant radicalement les coûts de fabrication. En même temps, ils doivent veiller à assurer la conformité du livre aux prescriptions religieuses. Jusqu'où l'Écriture divine peut-elle être matériellement transformée en vue d'être commercialisée au meilleur prix? Cette interrogation est placée au cœur d'une réflexion sur les rapports entre Coran, marchandisation et authentification.

Le Coran - que l'arabe désigne par mushaf (volume) pour ne pas confondre la Révélation (qur'ân) avec son support, à l'inverse de la Bible - ne se revendique pas tant comme une Écriture «sainte» que comme une Écriture « divine ${ }^{2} »$ : il est, aux yeux des musulmans, le «Livre de Dieu» (kitâb allâb). Cette conception de la Révélation impose des contraintes spécifiques dans la reproduction et la diffusion du livre saint. Ladoption tardive de l'imprimerie dans le monde islamique, près de quatre siècles après son avènement en milieu chrétien en est l'illustration. Les difficultés techniques, la place économique importante occupée par la corporation des copistes et le prestige de l'écriture manuscrite sont décrits comme autant de freins à l'introduction de l'impression dans les pays musulmans ${ }^{3}$ (Pedersen 1984; Atiyeh 1995; Gdoura 1985). Mais la résistance à l'imprimerie a tenu principalement à des raisons religieuses et culturelles: l'élite des oulémas, docteurs de la loi, craignait que le Coran ne soit altéré par sa reproduction technique et que la standardisation de l'imprimé ne bouleverse le système de transmission du savoir au fondement de son autorité (Robinson 1993; Fawzi 1990). Dans le cadre de l'apprentissage coranique, l'écrit, subordonné à un ensemble de pratiques orales, est un aide-mémoire qui permet son incorporation correcte (Eickelman 1978; Hirschkind 2005). Jusqu'à aujourd'hui, la question de la reproduction et de la vente du livre saint divise les différents courants de l'islam. Pour les traditionalistes, le Coran serait un «bien inaliénable» au sens où Annette Weiner (1992) entend cette notion, soit un bien trop précieux pour la continuité du groupe pour être sacrifié dans un échange commercial sans que cela ne fasse scandale.

Néanmoins, force est de constater que le mushaf se décline sous des formes et des supports de plus en plus variés: le mushaf aux pages parfumées, le mushaf au stylo électronique audio, le mushaf en ligne, traduit en plusieurs langues, etc. Cette diversification des supports pose question: quelles répercussions a-t-elle sur la transmission d'un texte fondée en principe sur des règles strictes vouées à en préserver l'«authenticité» (sadiq - qui désigne littéralement la "conformité»- ou sahîh - traduit par «vrai, saint, valide»)? Quel rôle les autorités religieuses jouent-elles dans ce processus? Et à quoi le terme «autorités» réfere-t-il dans le contexte de la mondialisation de la vie religieuse et du développement des outils numériques? Derrière ces interrogations s'en profilent d'autres, plus concrètes: comment fabrique-t-on et commercialise-t-on un livre du Coran? Comment s'en établit le prix?

Ces questions se posent avec une acuité particulière au Maroc où la libéralisation politique et économique depuis le milieu des années 1990 est allée de pair avec l'émergence d'une nouvelle industrie du livre (Janjar 2006). À Casablanca, 
dans le quartier des Habous (Encadré 1), c'est le mushaf qui en est le moteur. En suivant étape par étape la fabrication de livres saints, cet article vise à étudier l'élaboration de leur valeur marchande. Plus précisément, il s'agit d'explorer comment les échanges dont le Coran fait l'objet au cours de ce processus modifient sa valeur essentielle - religieuse - en l'articulant à des valeurs esthétiques et économiques. La réflexion porte sur les façons dont se construit l'authenticité du livre du Coran en examinant la superposition (ou le conflit) des valeurs qui lui sont attachées. Dans la continuité de la réflexion engagée par Luc Boltanski et Arnaud Esquerre sur le rôle joué dans la construction de la réalité sociale par la formation des prix, j'entends le prix comme «le résultat de l'épreuve à laquelle est soumise une chose quand elle change de main» (2016: 21). La valeur, quant à elle, est ici définie "comme étant un dispositif de justification du prix» (ibid.) préalable à l'achat. Il s'agit d'examiner la tension entre le prix et la valeur telle qu'elle est mise en actes et en pratiques par les libraires et éditeurs du quartier des Habous. Pour mener à bien cette étude, j'ai suivi les éditeurs de livres saints dans leurs échanges avec les différents intervenants de la chaine de production aussi bien nationaux qu'internationaux (surtout moyen-orientaux), publics et privés - graphistes, imprimeurs, calligraphes et «experts religieux» (comme ils sont appelés).

\section{Fabriquer le mushaf à tout prix!}

\section{Le monde de l'édition aux Habous}

Les livres saints commercialisés dans les librairies des Habous proviennent majoritairement du Caire, de Beyrouth, de Damas et plus récemment de Riyad où ils sont produits par milliers d'exemplaires (Gonzalez-Quijano 2002 [1998]; Mermier 2005). Lexonération des droits de douane sur l'importation des «livres culturels» (expression employée par le ministère de la Culture pour désigner tous types de livres à l'exception des livres scolaires) diminue encore les frais. Le Maroc représente un marché attractif pour les éditeurs moyen-orientaux nombreux à se rendre dans le souk. Dans les librairies, les commerçants marocains, égyptiens, libanais ou encore syriens négocient les prix autour d'un thé. Suivant le nombre d'exemplaires du Coran achetés, la qualité matérielle (papier, encre, reliure, colle etc.), esthétique (dorures, style calligraphique, etc.) et didactique (mise en pages, couleurs), les libraires discutent jusqu'à obtenir une réduction qui les satisfasse. Le but est de vendre des modèles distinctifs au meilleur prix. Au Maroc, comme ailleurs dans le monde arabe, le prix du livre n'est pas fixe. Un ouvrage fabriqué au Liban ou ailleurs est vendu à un prix différent dans chaque pays en fonction du niveau de vie (Santini 2006). Ainsi, le même livre peut être vendu à des prix différents au Liban, en Égypte, au Maroc et même dans les différentes librairies des Habous. La vente du mushaf représente une part importante du chiffre d'affaires des librairies et constitue un enjeu essentiel pour leur réputation. 


\section{Encadré 1. Le quartier des Habous, centre du livre arabe}

Situé dans le centre-ville de Casablanca, le quartier des Habous se présente comme un souk composé de bazars proposant à la vente un éventail de produits, notamment des livres. On trouve en particulier le mushaf, les recueils des hadîths (récits rapportant les actes et les paroles du Prophète) et les livres de turâth (œuvres issues du fonds du patrimoine littéraire, linguistique et religieux arabe). Érigé durant le Protectorat, le quartier a été construit pour séparer les lieux de résidence européens des lieux de résidence marocains qui s'étendaient suite à l'arrivée de familles de négociants prospères en provenance principalement de Fès. C'est là que fut fondée au xII siècle l'université al-Qarawiyyîn, point névralgique à partir duquel s'est constituée la culture du livre dans la ville. À leur installation aux Habous, les familles fassies ont continué à s'inscrire dans cette activité intellectuelle. Elles ont établi des librairies, et par la suite étendu leur activité à l'édition. Avant elles, des Syro-libanais avaient fondé les premières imprimeries et librairies du royaume près des nouveaux établissements scolaires indépendants qui avaient eux aussi souhaité bénéficier des prix de location peu élevés. En effet, le quartier des Habous est ainsi nommé car les biens immobiliers qu'il renferme appartiennent à l'État, qui les affecte à des œuvres de bienfaisance ${ }^{4}$. Les plus anciennes et les plus importantes maisons d'édition et de distribution (en gros et au détail) de ce quartier telles que Dar Attakafa ("maison de la culture»), Dar el Kitab («maison du livre») et le Centre culturel arabe se situent à l'entrée du quartier sous les arcades du souk al-betrina ("le bazar des vitrines»). Elles concentrent les activités d'édition, de distribution et parfois d'impression. Les librairies plus récentes se situent quant à elles le long de deux artères centrales du souk al-medina qui s'est considérablement étendu depuis les années 2000. Aujourd'hui, le quartier regroupe près de soixante-dix librairies, le plus grand nombre au Maroc.

\section{Encadré 2. L'enquête}

Cette étude s'appuie sur une enquête de terrain menée au sein de la maison d'édition-distribution (en gros et au détail) Dar Attakafa. Travailler en tant que libraire durant plusieurs mois m'a permis d'identifier les acteurs essentiels du quartier des Habous - marchands, éditeurs, calligraphes, graphistes, imprimeurs, clercs - et de saisir leurs interactions. Néanmoins, la relation d'enquête a posé plusieurs difficultés. Parler le dialecte marocain et manier un peu l'arabe classique ont été des enjeux cruciaux pour me faire une place. Mon premier entretien avec Mohamed Kadiri, à la tête de la maison, a pris la forme d'un contrôle de connaissances linguistiques. Tout au long de notre échange, il n'a pas cessé de corriger mes fautes d'arabe. De leur côté, les employés se sont amusés durant des semaines à m'interroger sur la traduction de certains termes. C'est seulement après avoir démontré que j'étais soucieuse d'apprendre l'arabe qu'ils m'ont assigné une place dans la librairie et attribué un nouveau nom: "Fatima». Ils me disaient: «lci tu t'appelleras Fatima et dehors, les gens continueront à t'appeler Anouk». L'attribution de ce prénom musulman
(Fatima est la fille du Prophète) et l'apprentissage de l'arabe m'ont permis de ne plus être une étrangère à leurs yeux - pour un temps, du moins. La seconde difficulté de l'enquête était d'ordre confessionnel: travailler au sein de librairies islamiques en tant que non musulmane a parfois été une position difficile à tenir. Un jour, un de mes collègues déclara, en français: «Maintenant que tu étudies le livre et que tu travailles à Dar Attakafa, tu dois choisir l'islam ». J'ai expliqué que je ne le souhaitais pas. Cette réponse tranchée a soudainement interrompu le jeu auquel mes collègues et moi nous étions efforcés de jouer depuis des semaines, au cours desquelles j'ai eu accès aux transactions et effectué des entretiens. Ces deux difficultés ont été instructives à plusieurs égards. Elles m'ont permis de comprendre que la vente du mushaf est avant tout une affaire d'hommes qui démontrent une piété musulmane, véritable ou non. ॥ n'est d'ailleurs pas anodin que les libraires demandent à certains clients s'ils sont musulmans avant de leur vendre un exemplaire. Ainsi, les normes s'appliquent aussi bien aux vendeurs qu'aux acheteurs.

Aux Habous, on distingue trois types de structures : un petit nombre (cinq) de grandes maisons d'édition et de distribution (en gros et au détail); une vingtaine de boutiques semi-grossistes qui vendent aussi à l'unité; et une quarantaine de 
points de vente au détail. Ces deux derniers types de commerce peuvent cependant éditer des livres ponctuellement. Les détaillants, à la tête de petites boutiques (deux ou trois employés) au faible capital, se fournissent auprès des semigrossistes et éditeurs-distributeurs qui comptent entre cinq et quinze salariés. Les semi-grossistes s'approvisionnent, pour leur part, auprès des éditeurs-distributeurs des Habous, mais importent la majorité de leurs livres du Moyen-Orient. Les petites et moyennes structures sont spatialement organisées autour des éditeursdistributeurs, fondés majoritairement au lendemain de l'indépendance. Depuis 2000, une vingtaine de petites librairies se sont créées pour exploiter l'engouement pour le livre islamique. Cette dynamique a été facilitée par l'absence de cadre juridique et l'exonération d'impôts sur le livre culturel. Le Code de la propriété culturelle et artistique de 1971 concerne uniquement les domaines cinématographique et musical. L'article 1 du Code de la presse, inchangé depuis 1973, stipule: «L'imprimerie et la librairie sont libres.» L'éditeur doit simplement être inscrit au registre du commerce et avoir un numéro de patente. Ainsi, les libraires des Habous évoluent dans un système libéral peu contrôlé. Ils se livrent une bataille acharnée pour proposer au meilleur prix un mushaf caractérisé par une «belle» écriture - à la fois esthétique et lisible -, des enluminures bien définies et une couverture agrémentée de riches matériaux.

\section{Des «bisnessmen» du mushaf?}

Ces marchands n'ont pas forcément une bonne connaissance de l'islam ou du Coran. La plupart vendent le livre saint davantage par circonstance que par conviction religieuse. Les nouvelles librairies ont été fondées par d'anciens employés des commerces plus établis. Âgés d'une trentaine d'années en moyenne, ces jeunes hommes (quasiment aucune femme n'est propriétaire d'une librairie), communément appelés les "petits libraires", ont appris le métier auprès de leurs aînés. Comme eux, ils sont généralement originaires du quartier. Les parcours de Mohamed Kadiri et de Abdelkader Hamdani, l'un de ses anciens employés, sont à ce titre éloquents (Encadré 3).

\section{Encadré 3. Les parcours exemplaires de Mohamed Kadiri et d'Abdelkader Hamdani}

Mohamed Kadiri a commencé à vendre des livres à l'âge de 12 ans. À leur départ de Fès au début des années 1950, ses parents et lui se sont installés près du quartier des Habous. À cette époque, les nouveaux migrants syro-libanais fuyant les tensions de leur pays ont fondé les premiers établissements scolaires indépendants, permettant ainsi le développement local de structures d'impression et de distribution de livres scolaires aux Habous. Muni d'une charrette, Mohamed Kadiri allait de librairie en librairie, achetant des manuels scolaires et des livres de littérature en provenance du
Moyen-Orient qu'il revendait aux marchés au gros de Casablanca où continuent de s'approvisionner les commerçants venus des quatre coins du Maroc. II a pu accumuler un capital suffisant pour ouvrir sa propre boutique dans un local spacieux situé à un emplacement stratégique. Au milieu des années 1970, lorsque l'État, engagé dans la refondation du système scolaire, a fait appel aux professionnels du livre pour concevoir les premiers manuels, Mohamed Kadiri s'est positionné dans ce nouveau marché. Avoir successivement exercé les professions de marchand et de distributeur lui 
avait permis d'acquérir une fine connaissance de ce produit. En 1975, il s'est associé à M. Kitani et a fondé les éditions Dar Attakafa. À cette période, cinq maisons dominaient le marché lucratif du livre scolaire. Au fil des années, beaucoup de ces maisons ont progressivement orienté leur commerce vers le livre islamique. En 1999, Mohamed Kadiri et M. Kitani, âgés d'une cinquantaine d'années, ont conçu leur premier modèle de Coran écrit par un calligraphe.

Abdelkader Hamdani, quant à lui, a intégré la maison en 1996. II était un enfant du quartier à la recherche d'un travail. Mohamed Kadiri, en quête de personnel pour préparer la rentrée scolaire, l'a recruté. Âgé de 19 ans, Abdelkader Hamdani a commencé par effectuer des tâches simples telles que décharger les cartons de livres, les ranger sur les étagères, prendre les commandes, etc. Ayant démontré qu'il était volontaire et efficace, il s'est vu confier la gestion des stocks ou le renseignement des clients, et son salaire a augmenté de 200 à 500 dirhams par semaine. Fort de cette expérience, il a décidé, treize ans plus tard, à l'âge de 32 ans, de créer son entreprise: "J'ai vu Monsieur Kadiri se faire tant d'argent avec les livres, je me suis dit que je pouvais faire la même chose à mon compte». Lorsqu'un petit local situé à proximité de la libraire Dar Attakfa s'est libéré, Abdelkader Hamdani a annoncé son départ. Deux mois plus tard il s'est associé avec deux cousins pour ouvrir sa propre boutique. Grâce aux compétences acquises et aux relations déjà établies avec de nombreux clients du quartier, Abdelkader Hamdani a développé rapidement son commerce.

Les deux hommes sont devenus libraires à des époques différentes parce qu'ils ont su saisir des opportunités au moment où elles se présentaient. Le choix de vendre et d'éditer le livre saint s'inscrit dans cette adaptation aux évolutions du marché. Comme eux, beaucoup de libraires se décrivent avant tout comme des «bisnessmen». Cependant, ils revendiquent aussi d'être de "bons musulmans». Cette présentation de soi est à mettre en relation avec le rôle essentiel de la réputation dans les relations de clientélisme et les échanges commerciaux aux Habous. Dans ce quartier comme dans d'autres souks (Geertz 2003; Mermier et Peraldi 2011), la «bonne réputation» est définie en fonction de critères essentiellement religieux. Montrer qu'on respecte les préceptes et les interdits musulmans apporte la garantie de certaines qualités éthiques valorisées dans le commerce. Pour s'attirer la sympathie des clients et susciter la confiance des fournisseurs étrangers, les éditeurs-distributeurs mettent en place un éventail de pratiques de mise en scène de soi. L'un d'entre eux ne se sépare jamais de son chapelet dont il égrène les perles à longueur de journée; un autre quitte la librairie plus tôt le vendredi de sorte que ses voisins le voient se rendre à la mosquée. Les éditeurs-distributeurs fortunés comme Mohamed Kadiri font régulièrement don d'exemplaires de Coran à la mosquée. Aussi, ils s'acquittent du devoir de la zakat (aumône rituelle) en finançant la construction de lieux de culte ou d'hôpitaux. L'apparence (beaucoup portent la barbe longue), le style vestimentaire (ils sont généralement vêtus d'une tunique couvrant le corps à hauteur des genoux ou des chevilles) et l'exercice ostentatoire de pratiques religieuses (prier cinq fois par jour aux heures prescrites) participent aussi de cette mise en scène de la piété.

Être un «bon musulman» n'est pas suffisant pour prétendre occuper une place de choix dans le souk. Être reconnu comme un «bon marchand» est une qualité primordiale. Aux Habous, les bénéfices sont socialement et moralement possibles et scéniquement organisés. Les marchands à la tête des grosses structures 
font constamment la démonstration de leur piété et de leur richesse. Par exemple, Mohamed Kadiri ne se déplace qu'en voiture $4 \times 4$ dernier cri et a l'habitude de sortir des liasses de billets quand il donne quelques dirhams à ses employés pour aller lui chercher un thé. Ainsi, les enjeux religieux et économiques sont étroitement imbriqués dans le commerce du livre du Coran.

\section{$\grave{A}$ chaque maison son mushaf}

Les stratégies commerciales allient une recherche continuelle d'économies pour faire baisser les prix et une diversification de l'offre afin de proposer des livres adaptés aux différents budgets et usages. Pour fabriquer le mushaf, les marchands ont recours à différentes options, plus ou moins coûteuses en fonction de leurs ressources. Ceux dont le capital le permet passent commande auprès de collègues égyptiens, libanais et syriens d'éditions «exclusives», comme ils les qualifient. À partir d'un modèle de Coran choisi dans leur catalogue, ils indiquent les changements à apporter pour créer une édition singulière sur laquelle apparaîtra le nom de leur boutique. Ces modifications ont également pour objectif de «répondre aux besoins des clients", d'ordre esthétique ou pratique déclare Fouad Korrich ${ }^{5}$, à la tête de Dar el Ilm ("maison du savoir») (Cohen 2010). La création d'un mushaf distinctif passe principalement par la conception de couvertures originales. Pour les modèles haut de gamme, certains éditeurs font construire de beaux coffrets où le livre est rangé. De différentes formes - boîtes, maisons, bateaux, etc. -, ces coffrets sont généralement fabriqués en Égypte, au Liban ou en Syrie, de plus en plus concurrencés par la Malaisie et la Chine où sont aussi produits le lutrin, le tapis, ou le chapelet dont la vente est généralement associée au livre saint dans les librairies des Habous et d'ailleurs (El-Alaoui 2006). C'est aussi au Moyen-Orient que le texte coranique est majoritairement imprimé avant d'être importé au Maroc où le secteur souffre de lacunes sur le plan matériel et humain. En outre, soucieux de s'adapter aux besoins de leur public dont un nombre grandissant cherche à apprendre la parole divine, un nombre croissant de marchands conçoivent des modèles de Coran didactiques dont la composition typographique vise à faciliter la mémorisation des sourates (Cohen 2012). Ainsi, couvertures, enluminures, couleurs, format et mise en pages occupent une place centrale dans la conception de modèles originaux.

Dans le but de diminuer le prix du livre, certains marchands, en particulier les petits libraires, réalisent eux-mêmes les compositions typographiques ou bien sous-traitent à un graphiste local. Dans les deux cas, ce travail consiste le plus souvent à scanner les pages d'un livre saint conçu au Moyen-Orient de façon à définir un nouvel encadrement et créer un «nouveau produit», dit Fouad Korrich. L'utilisation de logiciels comme Photoshop et In Design leur permet de changer facilement la mise en page, la taille et les couleurs des compositions végétales qui encadrent le texte ou encore d'importer des modèles d'arabesques prédéfinis. Enfin, il y a quelques éditeurs qui, comme Abdelmounim Soulami ${ }^{6}$, à la tête d'une 
imprimerie et d'une maison d'édition-distribution d'une quinzaine de salariés, ne souhaite pas faire commerce du livre saint: «Ce n'est pas n'importe quel livre, c'est le livre sacré! Je préfère ne pas y toucher». Il se refuse à le produire de crainte de voir ses «ouvriers marcher dessus, le déchirer» et que cela ne pose problème dans ses relations avec eux et/ou entre eux. Comme lui, un petit nombre d'éditeurs spécialisés dans le livre scolaire déplorent la marchandisation excessive du mushaf.

À travers la mise en place de différentes stratégies, la majorité des marchands cherche à concevoir le «best-seller» de demain. Ces tactiques se déploient également dans le domaine de la distribution. Les gros éditeurs-distributeurs cassent les prix en s'accordant des remises préférentielles: $30 \%$ contre $25 \%$ qu'ils accordent aux petits libraires, contraints de pratiquer des tarifs plus élevés. C'est pourquoi Fouad Korrich et d'autres petits libraires ont, un jour, tenté de boycotter les gros éditeurs-distributeurs. La nuit même, cependant, plus de la moitié d'entre eux se sont fait livrer en cachette. Mis au courant de la résolution prise par leurs clients et concurrents, les gros éditeurs-distributeurs avaient proposé à quelques-uns de leur accorder des remises plus avantageuses qu'aux autres s'ils acceptaient de se désolidariser du boycott. «Ils avaient juré sur le Coran!» dit Fouad Korrich, qui n'a d'autre choix que de continuer à traiter avec eux en leur achetant régulièrement plusieurs centaines d'exemplaires afin de proposer à ses clients tous les titres qu'ils recherchent. Ainsi, un libraire s'approvisionne auprès de ses concurrents qui deviennent donc aussi des partenaires et des clients. Cette interdépendance structure le monde de l'édition aux Habous.

La logique du profit n'est pas exempte, cependant, d'autres préoccupations, religieuses et sociales. Au souk des Habous, capital économique et capital symbolique, si souvent opposés dans la littérature (Duval et Garciat-Parpet 2012), fonctionnent de manière solidaire le long d'un continuum qui tend plutôt vers l'un ou vers l'autre, mais qui ne les dissocie jamais. Bien que l'islam interdise le ribâ (signifiant à la fois usure et intérêt), le mushaf est tout à la fois un «objet singulier»- défini par Lucien Karpik comme un bien multidimensionnel et de qualité incertaine pour lequel la concurrence par les prix est moins déterminante que la concurrence par la qualité (2007) - et un objet standard soumis aux lois du marché et de l'industrie. Ainsi, les éditeurs sont tenus de donner des gages d'authenticité en masquant la standardisation industrielle de la production du texte. Prêter attention à une pratique courante aux Habous permet de le comprendre. Comme de nombreux livres au Maroc et dans l'ensemble du monde arabe, le Coran est fréquemment "piraté», un mot que les marchands emploient eux-mêmes en français. Mais que signifie "pirater» dans le cas d'un livre qui représente la parole de Dieu? De quel original parle-t-on? Ces questions interrogent directement la construction de l'authenticité du livre saint et la manière dont les éditeurs se la représentent et la fabriquent. 


\section{«Pirater» l'Écriture}

\section{Le mushaf: un objet pluriel}

Alors que la Bible est soumise aux droits d'auteur (ou plutôt de traducteurs), le livre du Coran ne répond à aucune réglementation officielle. Ainsi que l'explique Bassam Kurdî̀, à la tête du Centre culturel arabe, maison d'édition-distribution maroco-libanaise d'une quinzaine de salariés spécialisée dans les ouvrages de sciences humaines et sociales: «Les droits d'auteur ça n'existe pas dans le Coran. Tu vas pas payer au Bon Dieu des droits d'auteur, l'auteur c'est qui? C'est le Bon Dieu, donc tu ne paies pas de droits d'auteurs». Il existe cependant des «droits de production et d'écriture» (huqûq al-kitâba) qui protègent les caractéristiques physiques du mushaf telles que les enluminures (tadhîb) ou encore la calligraphie (kbâtt) dans le cas où l'éditeur aurait fait appel à un calligraphe. Ces droits indiquent que l'originalité d'un Coran-livre réside dans ses propriétés matérielles. Au quartier des Habous, suivant le type de Coran vendu, majoritairement conforme à la lecture de Warsh ou à la lecture de Hafs - dont les variantes concernent des points de vocalisation, des emplacements de pause dans la récitation ou de fin de verset ainsi que des détails de prononciation ${ }^{8}$-, cette singularité repose davantage sur les ornementations ou l'écriture. Alors que les modèles de «Coran Hafs», comme les libraires les qualifient, sont majoritairement reproduits en «écriture d'imprimerie», de calligraphie naskhî (généralement adoptée pour les polices de caractères arabes), les modèles de «Coran Warsh» sont principalement imprimés en «écriture manuscrite» en calligraphie maghrîb̂ิ. Les expressions écriture « d'imprimerie» ou «manuscrite» sont en réalité trompeuses. En effet, la quasi-totalité des masahîf(pluriel de mushaf) produits dans le monde et importés au Maroc sont écrits à la main, scannés, retouchés puis imprimés après avoir été vérifiés et certifiés. C'est la manière que les producteurs ont trouvé pour préserver l'exactitude du Coran tout en exploitant les économies rendues possibles par les technologies numériques. Ce mode de fabrication - qui n'est pas sans rappeler la lithographie - permet de conserver les caractéristiques physiques d'anciens manuscrits comme le style calligraphique et les marques de vocalisation, qui soutiennent jusqu'à aujourd'hui la lecture et la récitation coraniques.

Les modèles de Coran Hafs à «l'écriture d'imprimerie» se conforment majoritairement à l'édition égyptienne dont ils reprennent le mode de lecture, les conventions d'imprimerie et les notes éditoriales contenues à la fin du volume (Albin 2005). Réalisée en 1924 au Caire sous le patronage du Roi Fouad I et d'Al-Azhar, première université d'étude islamique, cette édition est devenue l'équivalent d'une « édition officielle» (Azaiez 2013: 15), largement distribuée par l'Égypte, le Liban, la Syrie et l'Arabie saoudite où se trouve le plus grand nombre d'imprimeries de la région. Selon les éditeurs, ces modèles de Coran ne sont pas associés aux mêmes usages que les livres de Coran à «l'écriture manuscrite» de lecture Warsh, méthode de récitation la plus répandue au Maroc. Les premiers sont principalement achetés par des clients qui cherchent, selon les termes de Fouad Korrich, à les «offrir» 
ou à «décorer» leurs intérieurs avec de beaux modèles de Coran. «Comme ils ne les achètent pas pour lire, ils s'en fichent», poursuit-il. À l'exception de quelques clients, en particulier les jeunes, qui utilisent le Coran Hafs, plus lisible à cause de «l'écriture d'imprimerie» à laquelle ils se sont familiarisés à travers les manuels scolaires, les livres de littérature générale et les journaux, la grande majorité des clients recourt aux Corans Warsh en calligraphie maghrîbî.

C'est en effet ce modèle que les libraires des Habous déclarent vendre le plus, surtout aux personnes de plus de quarante ans ayant appris le Coran dans l'école coranique au moyen d'une planchette en bois. Cette méthode d'apprentissage, employée jusqu'à aujourd'hui dans les écoles coraniques qui accueillent plus de $88 \%$ des enfants âgés de 4 à 7 ans (Benghabrit-Remaoun et Bouzoubaa 2004), associe mémorisation, récitation et écriture (Fortier 2003). En outre, c'est ce modèle que les donateurs (principaux clients des marchands des Habous) achètent par centaines en vue de les offrir aux mosquées. Ainsi, deux types de marché s'organisent aux Habous: le marché du Coran à «l'écriture d'imprimerie» de lecture Hafs et le marché à «l'écriture manuscrite» de lecture Warsh.

La préférence d'une majorité de clients pour la lecture Warsh dans une gamme de prix autour de 20 dirhams pour un moyen format impose des contraintes spécifiques aux éditeurs-imprimeurs. Alors que la singularité du modèle de Coran à «l'écriture d'imprimerie» réside principalement dans les enluminures ou les matériaux, l'originalité des livres du Coran en calligraphie maghrîbî repose en revanche davantage sur l'écriture: "Elle est plus ou moins jolie, bien faite, lisible», déclare Fouad Korrich. Ces spécificités ont une incidence sur le dispositif de production des deux modèles de Coran et l'établissement de leur prix. Tandis que le coût du Coran Hafs est fixé essentiellement en fonction des matières premières (qualité de l'encre, du papier, de la couverture, etc.), celui du Coran Warsh est surtout calculé à partir de son style calligraphique.

\section{Le mushaf de Zwiten: un canon maghrébin?}

Pour concevoir un modèle de Coran Warsh, les éditeurs ont le choix entre deux options bien résumées par Bassam Kurdî: «Soit tu achètes les droits de celui qui a écrit le Coran, à lui [le calligraphe] ou à l'éditeur qui les lui a achetés, soit tu fais ton propre Coran en ramenant un calligraphe pour qu'il te fasse ton écriture, à toi». La dernière option consiste en un processus long et couteux que seules les éditeurs solides peuvent réaliser. Les petits éditeurs, au faible capital, piratent le mushaf, c'est-à-dire qu'ils reproduisent une édition sans avoir racheté les droits au calligraphe ou à l'éditeur. Â cette fin, ils scannent les pages du livre et en modifient les décorations à partir de logiciels de publication assistée par ordinateur. Ces transformations visent à concevoir un modèle différent de celui copié de manière à contourner les accusations de piratage. La version en calligraphie maghrîbî de lecture Warsh la plus fréquemment piratée est le «mushaf de Zwiten", comme il est communément appelé. Il s'agit d'une édition réalisée en 1929 par un célèbre faqî̉ (juriste) et calligraphe réputé pour son écriture et sa 
connaissance du Coran, dont les droits appartenaient jusqưà une période récente à la maison d'édition-distribution Dar al- mushaf al-sharif, située au Caire (Fawzi 1990). Nommé Zwiten, ce calligraphe était professeur à al-Qarawiyyîn, une importante université d'études islamiques à Fès. Selon Mohammed Maghraoui, spécialiste des arts traditionnels marocains et enseignant à l'université Mohammed $V$ à Rabat, cette édition est utilisée comme modèle car «il s'agit de la plus ancienne copie du Coran lithographiée avec la numérotation au Maroc ${ }^{9}$. C'est pourquoi, poursuit-il, le statut de Coran «authentique» lui a été conféré.Jusqu'à aujourd'hui, le «mushaf de Zwiten» est le modèle original des masahif le plus reproduit au Maroc par le scan ou la copie manuscrite. Il s'agit d'un livre canonique - différent de celui qui prévaut au Moyen-Orient.

Si la majorité des marchands des Habous piratent le modèle de Dar al- mushaf al-sharîf, certains ont créé leur propre édition en faisant appel aux services d'un calligraphe. Aujourd'hui, on dénombre une dizaine de copies calligraphiées et publiées par des éditeurs indépendants entre 1879 et 2006 (dont quatre sont parues ces quinze dernières années). Ces différentes éditions ont toutes été piratées, d'après Fouad Korrich, qui explique comment il est possible de reconnaître un Coran Warsh copié d'un original:

"On sait qu'il s'agit d'un livre piraté quand il ne dispose d'aucune indication: adresse, etc. Mais de toute façon, dès que tu vois cette écriture [lécriture maghrî̉î̀], tu sais que c'est piraté, personne ne se casse la tête à acheter les droits d'écriture ou à l'acheter non piraté parce que tout le monde sait que ce type de Coran est piraté. Si un éditeur est capable de te montrer un Coran sur lequel figure le nom de sa maison d'édition alors tu peux être sûr qu'il ne l'a pas piraté. Mais bon, ça m'étonnerait que tu en trouves beaucoup!» (Entretien réalisé le 20 février 2010 à Casablanca)

Les éditeurs qui réalisent ces copies scannées font apparaître sur la quatrième de couverture un autre nom que celui de leur maison de manière à brouiller les pistes et éviter toute possibilité de poursuites judiciaires. Néanmoins, celles-ci sont rares à cause des relations d'interdépendance entre les éditeurs. C'est pourquoi ils les redoutent peu et piratent le mushaf sans toujours prendre de précautions ${ }^{10}$. Bassam Kurdî explique comment «[son] Coran» a été piraté:

«Mes voisins l'ont reproduit et ils ne mont jamais racheté les droits d'écriture! Mais c'est pas grave je ne leur ai jamais fait de procès ni reproché quoi que soit. Le Coran n'est pas un produit commercial comme les autres. Je me dis: "après tout, c'est le Coran", si ça leur permet de le vendre et que plus de gens l'achètent, tant mieux, donc tu vois, c'est pas du piratage à proprement dit. Moi je ne fais pas de marges là dessus mais c'est pas grave. Je me dis qu'au moins ça fait plus de Coran, je ne te dis pas que je suis un prophète mais pour moi ça compte, c'est pour ça, je n'emmerde personne». (Entretien réalisé le 23 février 2010 à Casablanca)

La relation entre loriginal et la copie habituellement pensée en termes de dévaluation (Benjamin 2002 [1955]) est envisagée différemment par Bassam Kurdî. Pour lui, la copie n'altère pas l'original que son édition représente. Seule la perte économique 
compte à ses yeux. «L'aura» (pour reprendre les termes de Walter Benjamin) religieuse (et non esthétique telle que cet auteur l'évoque) de son édition, loin d'être compromise par les techniques illégales de reproduction modernes, serait même enrichie en devenant accessible à un plus grand nombre de lecteurs. Ainsi, selon cet éditeur, copier une édition du Coran, y compris la sienne, apparaît être une pratique presque légitime, du moins pas tout à fait illégale. C'est un exemple supplémentaire de la manière dont enjeux économiques et religieux s'entrecroisent pour enrôler les éditeurs et libraires dans leur propre auto-gouvernement. En interprétant leur profession comme une forme de culte, ils se convainquent que leur travail peut assurer le salut dans un autre monde. Daromir Rudnyckyj (2010) utilise la notion d' «économies spirituelles» pour référer à cette convergence du religieux et du capitalisme afin d'éclairer les affinités entre certaines pratiques religieuses et l'esprit du capitalisme ${ }^{11}$.

C'est donc principalement autour de deux «originaux» - le «Coran du Caire» et le «mushaf de Zwiten»-que le marché coranique s'organise au Maroc et plus globalement au Moyen-Orient. Derrière les distinctions éditoriales, en lien avec des traditions religieuses, calligraphiques et artistiques spécifiques, d'autres plus importantes, d'ordre politique voire idéologique se profilent entre ces pays. À titre d'exemple, l'Arabie saoudite (majoritairement hanbalite) est suspectée par la monarchie marocaine de vouloir propager $s a$ relation esthétique au texte révélé - et par là sa vision de l'islam - en distribuant gratuitement des milliers d'exemplaires de Coran fondés sur une matérialité (à la fois visuelle - style calligraphique - et sonore - méthode de lecture) différente de celle adoptée par le Maroc de rite malékite. C'est pourquoi, explique le directeur de la Fondation Mohamed VI pour l'édition du saint Coran, le roi a souhaité reprendre la main sur ce marché12.

En outre, au Maroc, comme ailleurs dans le monde musulman, c'est le primat de la forme manuscrite des éditions modernes du Coran qu'il est important de noter. La reproduction mécanique de l'écriture typographique a été largement remplacée par la reproduction mécanique de l'écriture manuscrite. Dans ce type d'édition, c'est donc par l'écrit que s'élabore «l'authentification » (c'est-à-dire l'exactitude) du livre saint. Celle-ci est construite non seulement par les éditeurs mais aussi, on va le voir, par les calligraphes et les «experts religieux».

\section{Le Coran «halal»}

\section{"Créer» son Écriture}

Dans le but de créer de beaux exemplaires de Coran et aussi d'être détenteurs de leur propre écriture, certains éditeurs des Habous ont demandé à un calligraphe (khâttat), on l'a dit, de réécrire le Coran. Bassam Kurdî revient sur les raisons de son initiative:

«Ca coûte très cher de faire recopier le Coran, ça prend deux ou trois ans, ensuite il faut trouver un bon khâttat, tu le paies 200000, 400000 dirhams, ça dépend, si c'est un truc 
qui est fait en deux ans, trois ans, quatre ans. C'est pas un truc que tu fais en une semaine: le Coran, tu l'écris lettre par lettre, c'est de la calligraphie, c'est du dessin, tu crois que tu peux écrire le Coran en un mois ou deux mois! C'est un travail qui prend des années donc tu dois évaluer le travail et payer le khâttat en conséquence. Mais tu peux aussi négocier et payer 10000 ou bien 20000 dirhams ce n'est pas un problème, mais ensuite, c'est long il faut le faire revérifier trois, quatre, cinq, six fois par des experts, c'est très long et très cher. Moi, j'ai fait mon propre Coran sur la base de celui égyptien [l'édition de Dar al-mushaf al-sharîf]». (Entretien réalisé le 23 février 2010 à Casablanca)

Toutefois, poursuit-il, cherchant à faire valoir la qualité de son produit, en ne manquant pas de grossir le trait:

«Je l'ai amélioré, j'ai corrigé toutes les erreurs. Pas les erreurs dans le texte: le Coran il n'y en a qu'un, c'est surtout les erreurs de calligraphie [par rapport aux règles de la calligraphie maghrîbî̀. J'ai allongé la lettre ici, je l'ai arrondie là. Et j'ai refait le décor moi-même, je l'ai rendu meilleur, puis je l'ai fait relire trois, quatre fois et quand j’ai été sûr qu'il n'y avait plus d'erreurs, je l'ai imprimé. Finalement, il y avait cinq erreurs que j'ai corrigées aux éditions suivantes. Ce sont les imams qui l'utilisaient qui sont venus me voir pour me dire: "Sibassam, le Coran que vous avez fait, il y a des erreurs là et là". Je leur ai demandé de me faire une liste des erreurs qu'ils avaient repérées et au moment où il a fallu le réimprimer, j’ai corrigé ma deuxième et troisième édition ». (Ibid.)

Ainsi, il peut être rentable pour un éditeur de créer sa "propre écriture » pour deux raisons principales: d'une part, il peut vendre son modèle à un meilleur prix suivant la beauté de l'écriture et la réputation du calligraphe, celle-ci étant principalement déterminée par les expositions auxquelles il participe. D'autre part, l'éditeur peut s'enorgueillir d'avoir «amélioré» le Coran, c'est-à-dire de l'avoir rendu plus conforme, ce qui constitue un précieux argument de vente auprès des donateurs, soucieux de distribuer des masahîf "garantis halal» (licites), comme disent les éditeurs. À cette fin, le choix des donateurs qui cherchent avant tout à recevoir les hasanât (bienfaits) de leur bonne action repose surtout sur la renommée d'une maison d'édition. Celle-ci leur est généralement recommandée par l'imam de la mosquée qu'ils fréquentent ou de celle qu'ils souhaitent approvisionner. C'est pourquoi Bassam Kurdî n’indique pas le nom de sa maison sur le mushaf qu'il édite: « $\mathrm{Si}$ je mets Centre culturel arabe sur mon Coran, il ne se vendra jamais parce que ma maison est spécialisée dans les sciences humaines et sociales». La préférence des clients va généralement aux entreprises égyptiennes, libanaises, syriennes ou saoudiennes spécialisées dans la production du livre saint. Leur ancienneté dans ce domaine apporte la garantie d'un mushaf produit dans le respect des règles. Cependant, les maisons moyen-orientales nont pas d'expertise dans la production du Coran Warsh en calligraphie maghrîbî. C'est pourquoi les clients doivent souvent se résoudre à acheter des masahîf conçus localement. 
Comme l'exactitude d'un Coran peut être mobilisée comme argument de vente par les éditeurs, elle peut aussi être utilisée comme moyen de négociation par les acheteurs. À ce propos, Bassam Kurdî rapporte une anecdote significative:

«Un mauritanien, imam dans une mosquée à Nouakchott, venait toujours m'acheter mon Coran par centaines: 500, 600 exemplaires. C'était un très bon client! Jusqu'au jour où il me dit: "Bassam, on m'a dit que ton Coran contenait des erreurs. Je ne peux plus te le racheter." J'étais très étonné, je l'avais fait vérifier par plusieurs oulémas puis corrigé. En fait, j'ai appris plus tard qu'il était allé à Dar Attakafa acheter leur modèle parce qu'il coûtait 0,5 dirham moins cher; du coup, j'ai baissé mon prix». (Ibid.)

\section{Le rôle des "experts religieux»}

Une fois le texte manuscrit finalisé, le marchand des Habous peut faire certifier son Coran par des "experts religieux", moyennant une rétribution financière. Mais il n'y est pas obligé. Le processus d'estampille du livre saint est en principe très strict. Suivant le protocole défini par l'université Al-Azhar dans les années 1920, un contrôle doit normalement être réalisé à chaque étape de production (Albin 2005). Mais en fonction de la réglementation propre à chaque pays, ce protocole est respecté de manière plus ou moins rigoureuse (ibid.). Contrairement à l'Égypte et à l'Arabie saoudite où les producteurs et les distributeurs de Coran sont tenus de faire contrôler leurs éditions par l'université Al-Azhar et le Complexe du Roi Fahd, au Maroc, c'est seulement depuis 2010 que l'État a mis en place une législation spécifique au livre saint.

Normalement, le processus d'estampille se décompose en trois phases. Pour un modèle écrit par un calligraphe, plusieurs mois peuvent être consacrés à la vérification du texte manuscrit par des oulémas spécialistes dans l'art de la récitation et dans les diverses méthodes de lecture. Encore appelés «correcteurs de Coran» (morajii al-mushaf), ces spécialistes doivent s'assurer de la conformité des éditions à la vulgate uthmanienne (du nom du calife Uthman). Une fois garantis que le texte est exempt de tout défaut, ils délivrent un certificat (shahâda) signé par chacun d'eux ${ }^{13}$. Par cette empreinte, ils se portent garants de l'exactitude de ladite édition. Les «correcteurs de Coran» participent activement à l'élaboration de la qualité d'un mushaf en assurant la croyance dans sa valeur. Néanmoins, il se peut que ce «certificat», figurant généralement sur la troisième de couverture, soit piraté par les éditeurs suivant les mêmes procédés que pour le texte coranique. Une fois la certification terminée, un nouveau contrôle doit normalement s'exercer à chaque phase de l'impression. Par souci économique, beaucoup d'éditeurs n'effectuent pas ces contrôles dans leur intégralité. Cependant, ils ont tout intérêt à le faire. «Cela vaut le coût. Ça lui [le Coran] donne plus de valeur sur le marché», remarque Bassam Kurdî. Il poursuit: «Des pays comme l'Arabie saoudite ou les Émirats arabes unis interdisent l'importation de copies non certifiées sur leur territoire». L'enjeu est donc de concevoir des modèles de Coran conformes et, ainsi, parvenir à les 
vendre sur des marchés particulièrement lucratifs. Aussi, il s'agit pour les éditeurs d'éviter la destruction de milliers d'exemplaires défectueux, ce qui représenterait une perte financière considérable et une atteinte à la réputation de leur maison.

Il existe plusieurs instances en charge de délivrer la shahâda. L'université AlAzhar en est une principale. Mais les éditeurs des Habous peuvent solliciter n’importe quel ministère des Affaires religieuses dans leur pays ou à l'étranger. Comme l'explique Bassam Kurdî:

«C'est comme un visa Schengen, tous les pays ne le donnent pas aussi facilement. En France par exemple, ça prend plus de temps, ils demandent plus de documents, alors les gens partent en Espagne ou en Italie. Pour le Coran, c'est pareil! Tu as plus de chances que ton Coran passe si tu le fais vérifier au Liban plutôt qu'en Arabie saoudite [où les contrôles sont plus strictes]». (Entretien réalisé le 23 février 2010 à Casablanca)

La valeur marchande d'un mushaf est en partie déterminée par la notoriété des experts qui en certifient la conformité. Comme les calligraphes, le prix de leur contribution varie suivant leur renommée, fondée sur deux qualités principales: l'institution religieuse à laquelle ils appartiennent et leur médiatisation sur internet et les chaînes de télévision qui les sollicitent pour offrir leur avis ${ }^{14}$. «Cette valeur nest pas la même si tu le fais faire par Al-Azhar qui est une sommité ou par le ministère des institutions islamiques de Djibouti!», déclare Bassam Kurdî. Le complexe du roi Fahd en Arabie saoudite est également cité par de nombreux éditeurs comme une instance de référence. Le prestige des deux institutions continue à leur conférer une centralité religieuse au Maroc. C'est surtout aux yeux des éditeurs que la réputation des «correcteurs de Coran» est capitale. Les clients, à l'exception de ceux qui font des dons aux mosquées, vérifient rarement le contenu de la shahâda avant un achat. Comme l'exprime Bassam Kurdî: «Les lecteurs s'en fichent, c'est plus pour moi, c'est par principe». Comme lui, beaucoup d'éditeurs cherchent à vendre un Coran «garanti halal», un label qui enrichit simultanément la valeur économique et symbolique de leur bien et de leur statut.

Néanmoins, pour des raisons pratiques et économiques, les éditeurs qui conçoivent leur propre modèle de Coran préfèrent s'adresser à des oulémas locaux plutôt qu'à des spécialistes d'instances prestigieuses, plus coûteux, pour vérifier leur édition. Il s'agit d'imams, d'universitaires ou de fidèles ordinaires qui revendiquent un accès direct aux textes sacrés et une connaissance reconnue de la lecture de Warsh et de la calligraphie maghrîbî. Dans un contexte où il n'y a pas de hiérarchie cléricale organisée, il revient aux oulémas de décider si une copie du Coran est licite ou non. Or, au Maroc, jusqu'à une période récente, le corps des oulémas était hétérogène, non hiérarchisé, et chacun de ses membres s'autorisait de son seul savoir pour juger en la matière, s'opposant parfois les uns aux autres. Pourtant, une instance publique de contrôle du Coran existait bel et bien, située à Rabat, la capitale du pays. Intitulée Dar el qur'an, elle avait été fondée sous le règne d'Hassan II 
au moment où le roi avait créé sa propre édition de Coran dans les années 1970. Mais les éditeurs nétaient pas tenus d'y avoir recours et, de fait, ils s'adressaient rarement à elle. Selon Mohamed Kadiri, cela tenait à une raison principale: «L'un des oulémas vit à Rabat, l'autre à Taroudant, l'autre à Marrakech, le temps qu'ils se mettent d'accord, j'aurai déjà produit et vendu mille Coran!» Enfin, beaucoup d'éditeurs des Habous ne connaissaient même pas l'existence de Dar el qur'an. Ces observations mènent à un constat important: avant la création de la Fondation Mohammed VI pour l'édition du saint Coran, les éditeurs n'avaient pas recours à l'État pour procéder aux vérifications de leurs modèles. Ils préféraient, pour cela, s'adresser à des oulémas étrangers ou «auto-proclamés». Ainsi, la parole religieuse nétait pas exclusivement portée par les clercs du Palais, mais se diffusait et se diluait, représentée par une diversité d'acteurs qui naviguaient dans un champ éminemment pluriel et concurrentiel.

\section{Conclusion}

Suivre la chaîne de fabrication du mushaf a non seulement permis d'étudier les pratiques de production spécifiques destinées à l'authentifier mais aussi à analyser comment, lors de sa transformation en livre, la valeur religieuse du texte se télescope avec d'autres valeurs, esthétiques et économiques qui, en retour, la modifient. La valeur marchande et symbolique du livre du Coran fluctue au gré des stratégies éditoriales visant à agir sur son aspect et son prix et en fonction des pratiques de piratage et d'authentification. Ainsi, mettre la question de la valeur dans sa relation au prix au centre de l'étude a fait apparaître les liens complexes entre les qualités religieuses et économiques du texte révélé dans l'organisation de ce marché particulier. La valeur religieuse est ainsi sans cesse mise en jeu par les éditeurs et fait l'objet d'arbitrages constants pour assurer la rentabilité d'une activité qui doit garder l'apparence de l'authenticité. En outre, cette démarche a montré de quelles manières ces qualités variaient suivant les choix des éditeurs et des acteurs avec lesquels ils coopèrent.

Enfin, porter attention aux rapports entre Coran, marchandisation, culture industrielle et authentification a mis en évidence de quelles façons l'asymétrie entre producteurs et usagers du livre saint se réduit considérablement. Avant 2010, l'éditeur jouait un rôle crucial dans le rapport que les fidèles entretenaient avec le Coran. Ses choix de mise en page, par exemple, influençaient directement les modes de son apprentissage. Aussi, pour certifier «son Coran», l'éditeur, prioritairement intéressé par la recherche de bas coûts, était libre de se référer à n'importe quel «expert», local ou étranger. Ainsi, le champ religieux de l'autorité dépassait le cadre traditionnel de ses agents au Maroc où s'est opérée une multiplication des sources d'autorité qui a définitivement brouillé le message du roi, «Commandeur des croyants». 
Cette remise en cause de l'autorité monarchique a provoqué la nationalisation des chaînes de production et de distribution du Coran en 2010. Pour préserver le rite malékite, Mohamed VI a aussi créé une édition nationale du Coran de lecture Warsh en écriture maghrîbî. Ces mesures s'inscrivent dans le contexte plus général de la réorganisation de l'islam marocain, initiée à la suite des attentats de Casablanca en 2003 dans le but «de lutter contre les extrémismes» (discours du trône le 30 juillet 2003). Le salafisme, courant proche du wahhabisme saoudien revendiquant un retour à l'islam des origines, est particulièrement visé par cette nouvelle politique religieuse dont le livre du Coran représente un instrument majeur ${ }^{15}$.

\section{Ouvrages cités}

Adelкhah, Fariba. 1998. Etre moderne en Iran. Paris, Karthala.

Albin, Michael. 2005. «Printing of the Qur'ān», in Encyclopaedia of the Qur'ān. Brill Online.

Amir-Moezzi, Mohamed (dir.). 2007. Dictionnaire du Coran. Paris, Robert Laffont.

Aтryen, Georges. 1995. «The Book in the Modern Arab World: the Cases of Lebanon and Egypt», in Georges Atiyeh (dir.), The Book in the Islamic World. The Written Word and Communication in the Middle East. State University of New York Press, The Library of Congress: 233-236.

Azaiez, Mehdi. 2013. «Introduction», in Mehdi Azaiez (dir.), Le Coran. Nouvelles approches. Paris, CNRS Éditions: 13-35.

Benghabrit-Remaoun, Nouria et Khadija BouzoubaA. 2004. «L'éducation préscolaire au Maroc et en Algérie », Perspectives, n 132 : 1-12.

Benjamin, Walter. 2002 [1955]. L'œuvre d'art à l'époque de sa reproductibilité technique. Paris, Payot.

Blachère, Régis. 2001. Introduction au Coran. Paris, Maisonneuve \& Larose.

Boltanski, Luc et Arnaud Esquerre. 2016, "L'énigmatique réalité des prix », Sociologie, $\mathrm{n}^{\circ}$ 7: 41-58.

Brockett, Alan. 1984. «Studies in Two Transmissions of the Qur'ân ", thèse de philosophie, université de St Andrews. URL: http://fr.scribd.com/doc/21972348/Studiesin-Two-Transmissions-of-the-Qur-an-byAdrian-Alan-Brockett.

Cohen, Anouk. 2010. «Le livre du Coran à Casablanca et à Rabat», Archives des sciences sociales des religions, $\mathrm{n}^{\circ}$ 150: 175-195.

- 2012. «Le Coran et ses multiples formes (Casablanca, Maroc)», Terrain, n ${ }^{\circ} 59$ : 70-87.

- 2015. «Léconomie du livre saint dans le Maroc contemporain", Cabiers du CAP, $\mathrm{n}^{\circ} 1$ : 73-102.

- 2016. Fabriquer le livre au Maroc. Paris, Karthala (Terres et gens d'islam).

Duval, Julien et Marie-France GarciaParpet. 2012. «Les enjeux symboliques des échanges économiques », Revue française de socio-économie, $\mathrm{n}^{\circ}$ 10: 13-28.

Eickelman, Dale. 1978. «The Art of Memory: Islamic Education and its Social Reproduction ", Comparative Studies in Society and History, vol. 20, $\mathrm{n}^{\circ} 4$ : 485-516.

El-Alaoui, Soraya. 2006. Les réseaux du livre islamique. Parcours parisiens. Paris, CNRS Éditions.

FAwzi, Abdulrazak. 1990. «The Kingdom of the Book: the History of Printing as an Agency of Change in Morocco between 1865 and 1912 ", thèse d'histoire, université de Boston. 
Fortier, Corinne. 2003. «Une pédagogie coranique. Modes de transmission des savoirs islamiques (Mauritanie) ", Cahiers d'études africaines, $\mathrm{n}^{\circ}$ 169-170: 235-260.

Gooura, Wahid. 1985. Les débuts de l'imprimerie arabe à Istanbul et en Syrie: évolution de l'environnement culturel (17061787). Tunis, Publications de l'institut supérieur de documentation.

Geertz, Clifford. 2003. Le souk de Séfrou. Sur l'économie de bazar, trad. par Daniel Cefaï. Paris, Bouchène.

Gonzalez-Quijano, Yves. 2002 [1998]. Les gens du livre. Édition et champ intellectuel dans l'Égypte républicaine. Paris, CNRS Éditions.

Haenni, Patrick. 2005. L'Islam de marché. L'autre révolution conservatrice. Paris, Seuil.

Hirsch KInd, Charles. 2005. "Media and the Qur'ân", in Encyclopaedia of the Qư'àn. Brill Online.

JANJAR, Mohammed-Sghir. 2006. «L'édition dans le Maroc indépendant: 19552003 ", Rapport thématique: Dimensions artistiques, culturelles et spirituelles, 50 ans de développement humain. Perspectives 2025, Casablanca: 43-61.

Karpik, Lucien. 2007. Léconomie des singularités. Paris, Gallimard.

Mermier, Franck. 2005. Le livre et la ville. Beyrouth et l'édition arabe. Arles, Sindbad et Actes Sud.
Mermier, Franck et Michel Peraldi (dir.). 2011. Mondes et places du marché en Méditerranée. Formes sociales et spatiales de l'échange. Rabat / Paris / Beyrouth, Karthala / Centre Jacques Berque / IFPO.

NJoto-Feillard, Gwenaël. 2012. L'Islam et la réinvention du capitalisme en Indonésie. Paris, Karthala.

Pedersen, Johannes. 1984. The Arabic Book. Princeton, Princeton University Press.

Peters, Ruud. 2005 [1960]. «Waqf», in Encyclopédie de l'islam, t. XI. Leiden, Brill et Brill Online.

Prémare, Alfred-Louis De. 2004. Aux origines du Coran. Questions d'bier, approches d'aujourd'hui. Paris, Téraèdre.

Robinson, Francis. 1993. «Technology and Religious Change: Islam and the Impact of Print», Modern Asian Studies, 27 (1): 229-251.

Rudnyckyj, Daromir. 2010. Spiritual economies. Ithaca, Cornell University Press.

Santini, Maud. 2006. Paris, librairie arabe. Marseille et Aix-en-Provence, Parenthèses et MMSH.

WEIL, Laurent. 2014. «Éditorial. Finance islamique, éthique et rémunération ", Les cabiers de la finance islamique, $\mathrm{n}^{\circ}$ 6: 3-6.

Weiner, Annette. 1992. Inalienable Possessions. Berkeley, University of California Press.

\section{Notes}

1. Décret sur la création de la Fondation Mohammed VI pour l'édition du saint Coran (23 février 2010). Depuis sa création en 2010, la fondation élabore des statistiques sur la production et l'importation du Coran.

2. "Cette revendication s'exprime, au niveau théologique, par l'affirmation du tanzîl, la "descente", conçue comme mode opératoire de la révélation du livre. Le messager de cette révélation, reçue par l'intermédiaire de l'ange Gabriel, n'en est que le transmetteur passif; il n'a aucune part active à ce qui serait son élaboration, et à plus forte raison, à l'expression de son contenu» (Prémare 2004 : 27).
3. L'impression généralisée du Coran n'a pas commencé avant que la technique lithographique ne devienne disponible dans la région. Inventée à la fin du XviII ${ }^{\mathrm{e}}$ siècle en Allemagne, cette méthode d'impression présentait des avantages par rapport à la typographie. Elle permettait notamment la préparation calligraphique du texte coranique, un moyen d'assurer son exactitude fondée sur les versions manuscrites (Albin 2005).

4. À la recherche de la définition du mot Habous dans l'Encyclopédie de l'islam, le lecteur est renvoyé à celle de waqf: «En droit musulman, l'acte de fondation d'une institution charitable, d'où l'institution 
elle-même. L'essentiel pour quiconque a l'intention d'accomplir une pieuse action est qu'il ou elle déclare qu'une partie de ses biens immobiliers est désormais inaliénable et qu'il ou elle désigne des personnes ou des services publics comme bénéficiaires de leurs revenus» (Peters 2005 [1960]).

5. Âgé d'une quarantaine d'années, Fouad Korrich a succédé à son père en 2006. Fondée en 1955, Dar el Ilm a été l'une des premières librairies du quartier. À la différence de ses confrères, il ne s'était pas familiarisé avec les métiers du livre avant de prendre la direction de la société familiale. Ce qui explique pourquoi, selon de nombreux libraires, elle a fait faillite en 2009. Fouad Korrich est retourné à son activité initiale en tant que responsable commercial d'une compagnie de télécommunications à Casablanca.

6. Âgé de 37 ans, Abdelmounim Soulami a repris la société de son père il y a une quinzaine d'années. Fondée au milieu des années 1950, Dar Soulami figure parmi les plus anciennes librairies du quartier. En 2006, Soulami fonde l'imprimerie Imarsi et nomme sa sœur à la tête de la maison d'édition-distribution.

7. C'est en 1958, après son départ du Liban que le beau-frère de Bassam Kurdî fonda la librairie dans le quartier des Habous. Dès l'âge de 15 ans, Bassam Kurdî y a travaillé chaque été à la caisse. Las des études, il s'est impliqué davantage dans les activités du commerce où on lui a confié plus de responsabilités. En 1978, la maison de distribution a doublé ses activités d'une spécialité éditoriale. Aujourd'hui, le Centre culturel arabe est installé entre Casablanca et Beyrouth.

8. Hafs et Warsh sont les appellations données à deux des sept lectures coraniques (qirẩa). «Il s'agit de façons différentes de rendre (réciter) le texte initialement par oral, par la suite aussi par écrit [...] Trois variantes s'imposèrent au fil des temps, celle de Nâfi' transmise par Warsh, celle de 'Âsim transmise par Hafs et celle de Abû 'Amr transmise par al-Durri» (Amir-Moezzi 2007 : 814). Aujourd'hui, à l'exception de larges territoires d'Afrique occidentale et centrale où la lecture de Warsh, généralement associée au rite malékite, est répandue, c'est la lecture de Hafs qui, dans le reste du monde musulman, est la plus connue. Voir Blachère (2001) et Brockett (1984).

9. Propos recueillis lors d'un entretien réalisé le 8 décembre 2013 à Rabat.

10. Il arrive que des éditions soient reproduites avec des erreurs principalement liées aux conditions techniques d'impression - pages manquantes ou désordonnées - qui portent atteinte à l'exactitude du texte. De nombreux clients dénoncent ces pratiques ainsi que les livres du Coran "frauduleux» acquis dans les boutiques. À ce sujet, voir Cohen (2015).

11. Sur la marchandisation du champ religieux en milieu islamique, voir Haenni (2005); Adelkhah (1998); Njoto-Feillard (2012).

12. Entretien réalisé avec le directeur de la Fondation Mohammed VI pour l'édition du Saint Coran le 15 avril 2013 à Mohammedia.

13. La shahâda fournit une description de la nouvelle édition (sa taille et style calligraphique) et précise qu'en cas d'inexactitude, les copies seront confisquées.

14. Sur ce point, il est intéressant d'établir une comparaison avec les «Sharia Scholars» qui certifient la conformité des activités financières des banques islamiques. Ici aussi, la réputation de l'expert détermine le prix de la certification. Les plus connus peuvent toucher $1500 \$$ par heure de consultation en plus d'un bonus annuel compris entre 100000 et 200000 \$ par place sur un Sharia board (Weil 2014).

15. Un article en cours de préparation examine le processus de nationalisation du "Coran marocain». A travers l'étude de sa fabrication, il analyse comment les spécificités physiques du livre sont mobilisées par la monarchie pour créer un nouveau registre sensoriel afin de mieux encadrer le rapport sensible des fidèles au texte, en particulier sa transmission. 